\title{
Current approaches and novel treatment methods for cancer and radiotherapy
}

\begin{abstract}
Cancer is a type of illness caused by abnormal growth of the cells in the body. The cells reproduce uncontrollably and cause metastases in the body. The accumulation of these cells causes the tumor to be benign and malignant tumors. Radiation therapy is one of the major subsets of medical physics. Radiation therapy is a method for treating cancer that destroys cancer cells by high-energy radiation or preventing cancer cells from spreading. The rays used to treat cancer are $\mathrm{X}$-rays, gamma rays and charged particles. Radiation therapy is done in two ways, out of the body and inside the body. Radiation therapy, in addition to affecting cancer cells, also affects healthy cells. Therefore, methods should be used to keep healthy cells exposed to radiation. Conventional radiotherapy uses simulations of two-dimensional therapy and simple collimator but modern radiotherapy uses 3DCRT (Three Dimensional Conformal Radiation Therapy) and IMRT (Intensity-Modulated Radiation Therapy) from Multileaf Collimator and advanced simulation software. This article briefly reviews some of the new ways of treating cancer, such as immuno-oncology, drug delivery, and hyperthermia.
\end{abstract}

Keywords: cancer treatment, radiation therapy, X-rays, oncology, drug delivery
Volume 4 Issue 5 - 2018

\author{
Hamidreza Shirzadfar, Mahtab Khanahmadi \\ Department of Biomedical Engineering, Sheikhbahaee University, \\ Iran
}

Correspondence: Hamidreza Shirzadfar, Department of Biomedical Engineering, Sheikhbahaee University, Isfahan, Iran, Email h.shirzadfar@shbu.ac.ir, h.shirzadfar@gmail.com

Received: June 27, 2018 | Published: October 03, 2018

\section{Introduction}

The cells of the body each have DNA that causes reproduction and growth in the body. Cancer occurs when the cells exit their normal state and abnormally multiply. Consequently, they damage the adjacent tissues and ultimately become tumors. Cancer cells with circulatory and lymphatic system transfer to other parts of the body and cause the whole body to become infected with cancerous cells. Approximately over 200 types of cancer are known, each of which has its own side effects. ${ }^{1}$ Common cancers in women include breast, lung and cervical cancer. Gastric, prostate, and liver cancers are also common in men. ${ }^{2}$ Cancer in older people increases more because stem cells lose their defensive power. Some inappropriate nutrition and health patterns, air pollution, exposure to harmful rays, stress and obesity can cause cancer, and also about 5 to 10 percent of cancers are hereditary. Cancer is one of the most important causes of death in the world. Cancer can be cured or reduced its growth rate by various methods such as surgery, radiotherapy, Chemotherapy and gene therapy. ${ }^{3,4}$ The history of radiotherapy is the discovery of $\mathrm{x}$-rays that, when exposed to radiation, causes burns, so physicians used this method to treat skin diseases such as lupus and chronic skin ulcers. With further research, they discovered that these beams have antibacterial properties that were used for wound healing tuberculosis. They then used more energy beams to treat cancer of the internal organs of the body. ${ }^{5}$ In radiation therapy, using a linear accelerator, the electrons hit to the tungsten target, which causes the formation of $\mathrm{X}$-ray that produces high-energy ions in the body that destroys cancer cells. Radiation therapy uses X-rays, gamma, alpha and beta. These rays damage the DNA of the cancer cell and cause the cell to not grow and disappear. In this way, healthy cells also get damaged, but after a while, most of these cells are restored. Radiation therapy is used to kill cancer cells, improve organs such as the liver, kidneys and prostate, as well as prevent the spread of tumors and cancerous tissues. Radiation therapy is divided into three parts: internal (brachytherapy), external and systemic. ${ }^{6}$ MRI (Magnetic resonance imaging), CT scan (computed tomography scan), PET (positron emission tomography) and so on are used to identify cancerous tissues. In radiation therapy, monitoring and control of radiation dose is very important and should be set up with high precision, that is, it has the least damage to the healthy tissue and has the greatest damage to the cancerous tissue, which this is the task of the dosimetrist. The dose rate and the number of treatment sessions depend on the type of tumor, the location of the tumor and the patient's condition. ${ }^{7,8}$ Radiation therapy is for radical therapy or for palliative. The palliative method is to reduce the pain caused by cancer as well as increase the patient's longevity. To treat various types of cancer and tumors, radiation therapy is used singly or with chemotherapy or surgery. Specialists in radiotherapy include oncology radiotherapy specialists, medical physicians and dosimetrist. In radiation therapy, there is a part called the CT Simulator that takes images from the patient's body, and the physician uses these images to design a therapeutic method. ${ }^{9}$ Cancer is one of the deadly diseases that has been the species of many different treatments for the disease. There are several factors that can cause cancer, which can be prevented by observing some of the issues. Cancer treatments are basically dangerous and harm healthy tissues in the body. Therefore, researchers are looking for methods that have the best performance and the least damage to healthy tissues. Further, all types of cancer treatment and radiotherapy are reviewed.

\section{Materials and methods}

In the following, the paper explores the methods of radiation therapy.

\section{External radiotherapy}

In this method, the radiation therapy device outside the patient's body transfers ionizing radiation into the target tissue. In this way, the radioactive substance is not injected into the body, so the patient does not store in his body and does not emit radiation from his body and can communicate with others. This method is carried out over a period of several days or several weeks. This method does not require hospitalization. This method is used to reduce the metastatic pain and 
complications of the spread of the tumor. External radiotherapy is used for a variety of cancers such as brain, bladder, breast. ${ }^{10}$

\section{Brachytherapy}

In this type of method, the seeds or tubes that contain the radioactive substance are placed temporarily or permanently within the body that these radioactive substances are placed inside or near the cancerous tissue. In this way, the radioactive substance is implanted in the body. Implants used in this method are in shape, bullet, capsule, needle, granular or tubular. In internal radiotherapy, the patient is hospitalized and anesthetized in an isolated room, and according to the type of cancer, an implant containing a radioactive substance is placed in the body by an applicator or catheter (Figure 1). In the temporary method, the patient must be hospitalized while the radioactive substance is in the body and other people avoid them because the patient emits radiation. In the permanent way, the first few days when the radioactive substance has the most activity, the patient should be away from other people, but after a few days when the effect is less, the patient can leave the hospital. ${ }^{11}$ Brachytherapy is performed with High-Dose-Rate (HDR) and Low-Dose-Rate (LDR). In internal radiotherapy, radioactive isotopes such as Phosphorus, Phosphate, Cesium and Palladium are used. This method is most commonly used for cervical and prostate cancers. ${ }^{12}$ In systemic radiotherapy, the radioactive substance is also fluidly injected into the patient's body as a drink or injection, which is most commonly used for thyroid cancer and non-Hodgkin's lymphoma. ${ }^{13,14}$

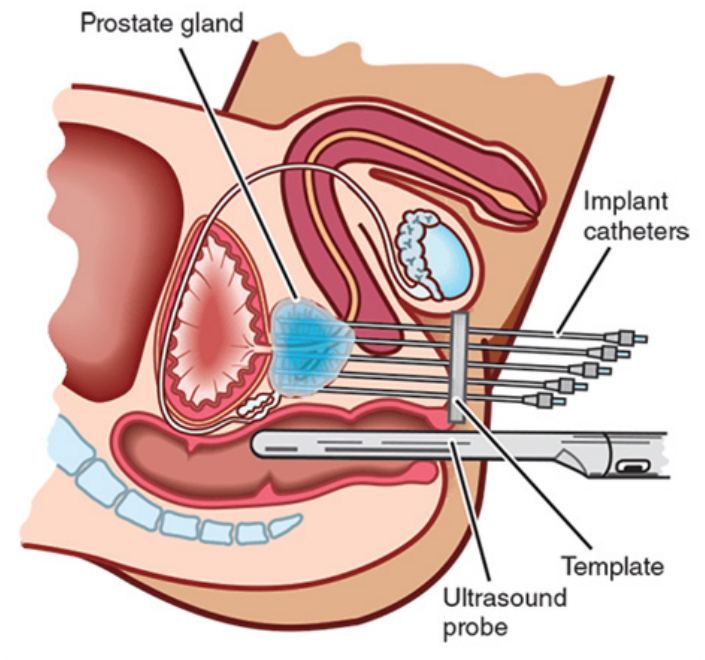

Figure I Brachytherapy for prostate cancer. ${ }^{14}$

\section{Radiation therapy simulation}

Before the radiation therapy, the patient's treatment should be designed. In simulation, the exact location of the tumor and the cancerous tissues as well as the thickness of the tumor is determined so that radiation can be done with high precision and healthy tissues are less damaged. The physician and physicist have tasks that include determining the exact location of the cancer tissue, calculating radiation dose and how it is done. CT simulator is used to simulate the treatment, which includes a CT scan, positioning lasers, marking system and 3D therapeutic design software. ${ }^{15}$ In this method, CT, MRI, PET and SPECT are used. In CT Simulator, the patient needs to be completely stable and requires the contrast agent and placement of the marker on the patient's body. The placement of beams on the target tissue is simulated by the software. In this method, the patient is fixed on the bed by using Mold or other tools, and then the simulation is performed and the target location is characterized by permanent or temporary markers. Radiotherapy design is done in two-dimensional and three-dimensional form. The two-dimensional method is an elementary method that only measures width and height. But, in $3 \mathrm{D}$, the width, height, and depth are determined. ${ }^{16}$

\section{Three dimensional conformal radiation therapy (3DCRT)}

In this way, the design is a three-dimensional treatment and is used for advanced simulation software and computers. In this method, the tumor is positioned with high precision, so the healthy tissue around cancer cells and tumors is far from radiation, and a higher dose can be used to destroy the tumor. This method is most commonly used to treat nasopharyngeal and prostate and liver tumors. In this radiotherapy using the CT scan, the target tissue is simulated virtualistically. This simulation is stored as a digital file on computers and the radiotherapy team can access this file. The physician can then design the treatment with advanced software and check out the tumor position in three dimensions (Figure 2). By this method, the intensity of radiation to the cancerous tissue can be determined with higher precision. This information is stored in the computer and is available to the radiation therapy team. Oncologist specifies a specific treatment for each patient, and the dosimetry and physicist regulate the radiation dose and type of beam. In this method, a virtual simulation session is performed and then the target points are identified with colored tattoos on the patient's body. ${ }^{17-19}$
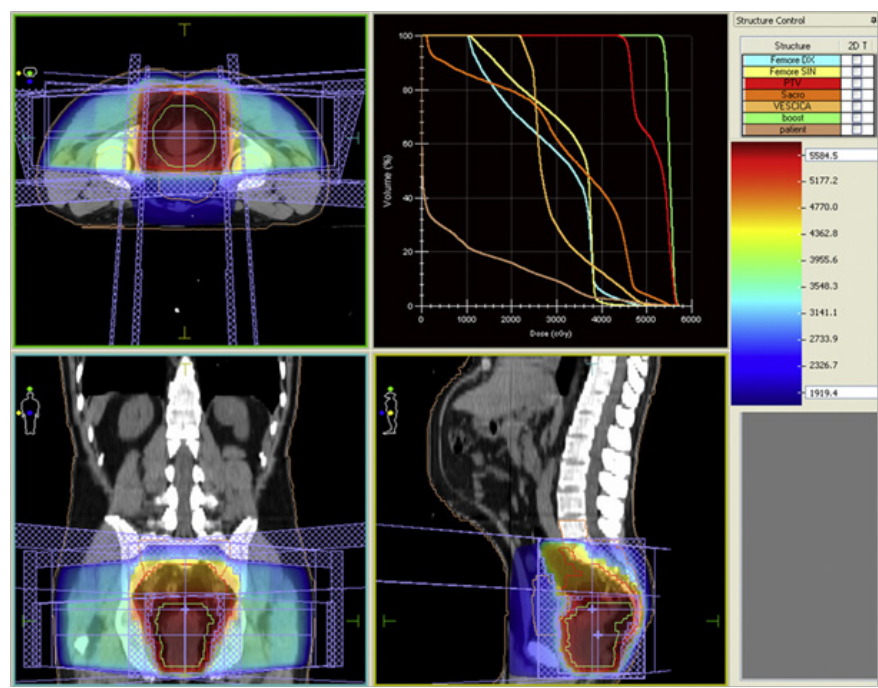

Figure 2 Simulation of treatment by Method 3DCRT. ${ }^{19}$

\section{Intensity-modulated radiation therapy (IMRT)}

This kind of radiotherapy is a three-dimensional new method that uses X-rays of varying intensity at the same time for irradiation. This method has a Multileaf Collimator, which enables the beams to accurately radiate and formulate the beam accurately. This method is less harmful than other methods. The radiation tube rotates around the patient and the tumor is irradiated from different angles (Figure 3). The IMRT method is more complex and longer than 3DCRT. In this way, healthy cells are less irradiated than cancer cells. This method is most commonly used for brain, head and neck, and liver tumors, and is not applicable to all cancers (Figure 4). This method has been used less frequently in oncology centers, because it is highly specialized. $^{20-23}$ 


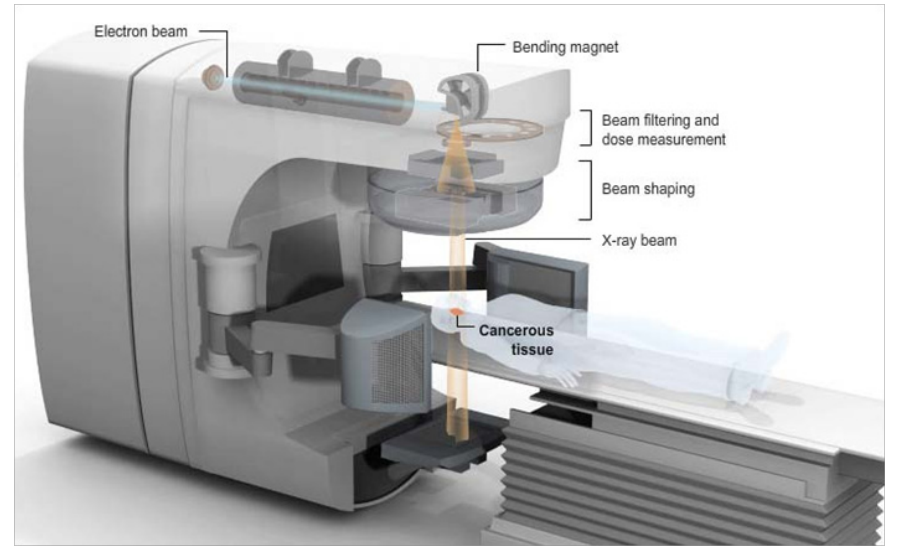

Figure 3 Intensity-modulated radiation therapy device. ${ }^{22}$

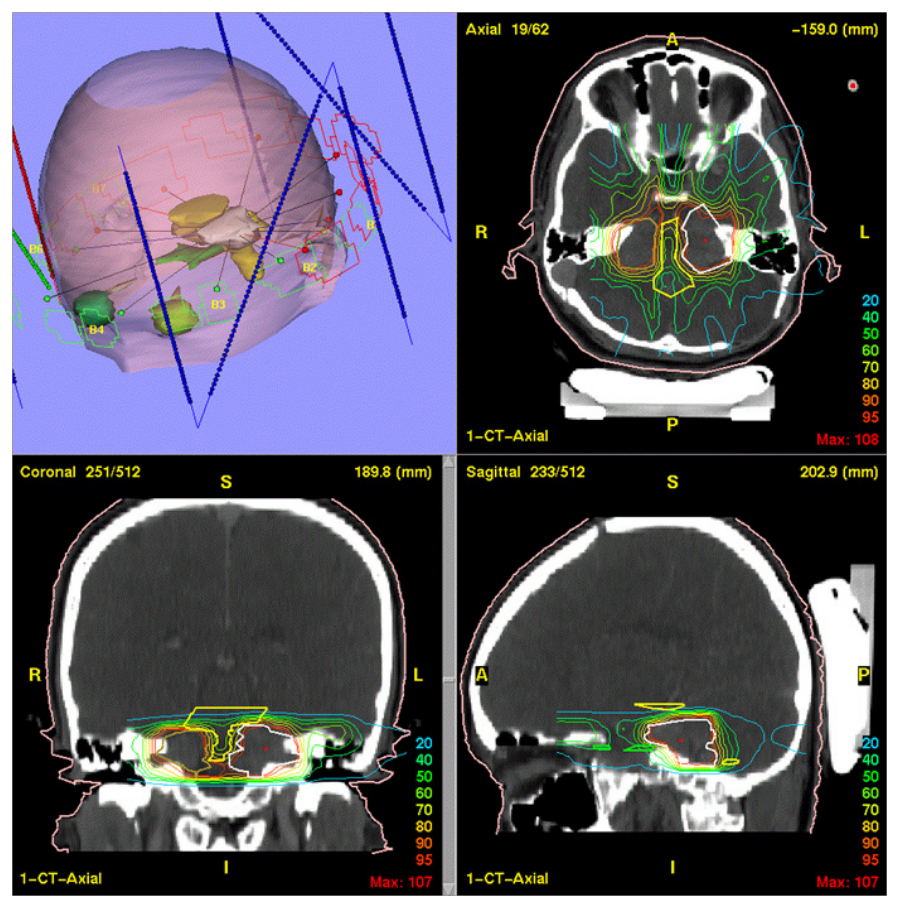

Figure 4 Simulation of treatment by method IMRT. ${ }^{23}$

\section{Stereotactic radiosurgery}

Stereotactic Radiosurgery is an advanced radiation therapy that is used for tumors that are inaccessible to the skull (Figure 5). In this method, a special cobalt 60 device or a linear accelerator or heavy charged particles such as helium is used. This method is based on an accurate positioning of the tumor location. In this method, the $\mathrm{X}$-ray tube rotates and irradiates around the target tissue. In this way, the cancerous tissue undergoes lethal radiation, but a healthy tissue will hit a smaller amount of radiation. This method is applicable to a variety of lesions and functional disorders (Figure 6). In this method, a high dose for irradiation is used. This method is applicable to hypophysis gland cancer, meningioma, or epilepsy and Parkinson's disease. Stereotactic Radiosurgery is a method for treating metastasis of the brain. ${ }^{24-27}$

\section{Radiation therapy equipment}

Radiation therapy devices that produce ionizing radiation destroy the DNA of tumor cells. These devices are divided into different types based on performance and energy, including: Orthovoltage, grenz ray, high voltage, contact, etc. The Ortho voltage device works at a voltage between $200-300 \mathrm{kV}$, which is less used due to some problems. The grenz ray has a minimum energy of about $20 \mathrm{KV}{ }^{28,29}$ High voltage devices include linear accelerators and cobalt 60 with a power of more than $1 \mathrm{MV}$. The linear accelerator accelerates electrons to the target with high energy. This device is less harmful to the skin than the Cobalt 60 device, and its radiant beams are more uniform. Cobalt 60 device uses a cobalt 60 source for irradiation. Cobalt is the best radioactive substance for cancer treatment. Cobalt produces gamma rays with energy of about $1.25 \mathrm{MeV}$, and can be used for deep tumors. This device is relatively old, causing burns to the skin. ${ }^{30,31}$

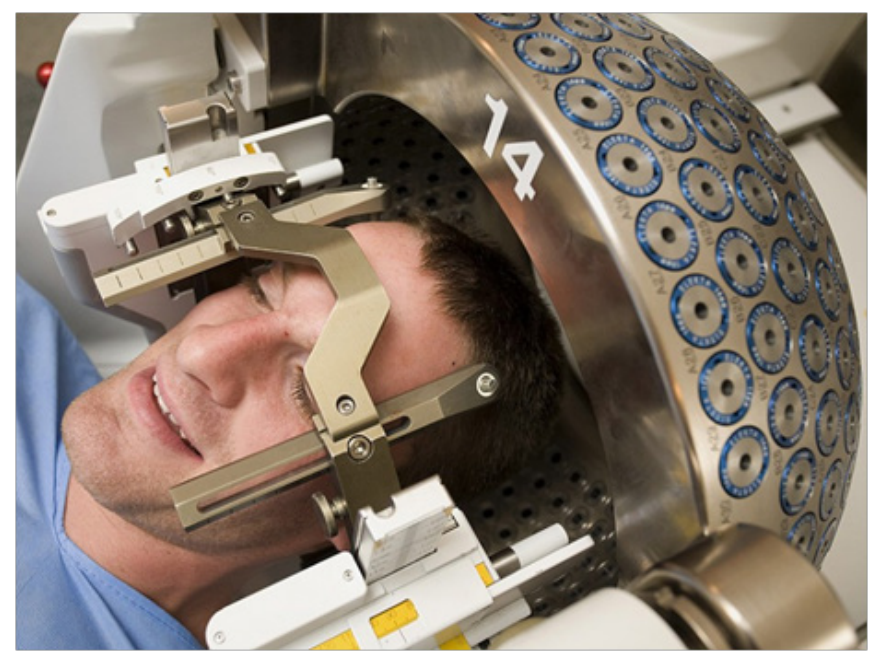

Figure 5 How to place the stereotactic radiosurgery device on the skull. ${ }^{26}$

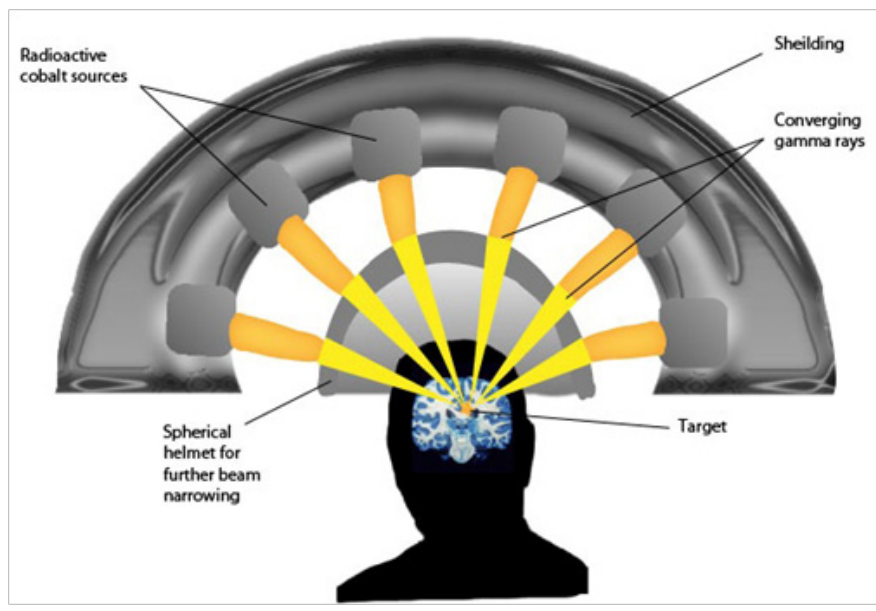

Figure 6 How to irradiate the tumor with stereotactic radiosurgery device. ${ }^{27}$

\section{Side effects of radiation therapy}

Radiation therapy has many complications from various psychological, physical and economic aspects. Psychologically, this method has depression, frustration, fatigue and discomfort. This method causes urinary disorders, lymphatic Edema, hair loss, skin changes, diarrhea, nausea, anorexia, joint discomfort and infection. Radiotherapy is costly due to the use of advanced devices and equipment, which is one of the problems for patients. In some cases, radiation from radiation can damage healthy cells, and this method is harmful to pregnant women and children._Radiation therapy 
for ovaries and testicles leads to infertility. Radiation therapy in breast cancer causes nerve damage, pain and swelling in the chest, respiratory problems, and irregular heartbeat. To reduce psychiatric complications during radiation therapy, the patient should be treated by a psychologist. It can also reduce these unpleasant effects with proper diet and exercise. ${ }^{32}$

\section{Cancer treatment methods}

The task of the immune system is to identify and destroy the unknown cells. One of the main causes of cancer is the immune system's inability to detect cancer cells because some of their cancerous cells hide themselves. So an Immuno-oncology technique was created that affects the immune system of the individual. This method is one of the newest therapies for cancer that destroys tumors and cancer cells by enhancing and stimulating the body's immune and defense system. Various factors, including inappropriate feeding, immobility and stress, can weaken the immune system. This method is very important in the treatment of breast, lung and digestive cancers. Immunosuppressive drugs have had a significant impact on skin cancers, such as melanoma. In this method, immune cells are removed from the body and cultured outside the body and injected again into the body. ${ }^{33}$ The Epigenetic method is another new way of treating cancer that changes the function of the gene and DNA. In this way, cancer cells are not destroyed but returned to their normal state. ${ }^{34}$ Another method is the use of a system called drug delivery. In this method, the drugs used to treat cancer are labeled by a nanoparticle, and then this nanoparticle enters the body and penetrates directly into the cancerous tissue and does not harm other healthy tissues (Figure 7). Magnetic nanoparticles are very suitable because of interacting with the external magnetic field, as well as controlling the movement of materials in the body. Magnetic nanoparticles do not damage other tissues of the body and are transported precisely to the target tissue. These nanoparticles can be used as a detector due to their magnetic properties. The magnetic property is due to the momentum of the electrons. These nanoparticles are used in medical sensors, chemotherapy and thermotherapy. One of the finest magnetic nanoparticles is iron oxide $\left(\mathrm{Fe}_{2} \mathrm{O}_{3}\right)$ because the production of this nanoparticle is easy and inexpensive, it is compatible with the internal environment of the body. ${ }^{35-37}$ Drug on magnetic nanoparticles in the form of ferro fluid is injected into the body. In the targeting step, labeled nanoparticles should enter the cancerous tissue and not damage other healthy cells. As a result, the nanoparticles target the cancerous cells and destroy the unhealthy tissue by drug. In the loading step of the drug, magnetic nanoparticles should be designed according to the drug in order to prevent non-specific cellular interactions. The nanoparticle should be able to protect the amount of drug that is carried out by the type of nanoparticle coating. In the targeting step, labeled nanoparticles should precisely enter the cancerous tissue and not damage healthy tissues.

The external magnetic field affects the bipolar atoms of the magnetic nanoparticles and drives the nanoparticles. As a result, magnetic nanoparticles are directed toward the cancerous cell, and the drug on these nanoparticles causes the cancer cell to disappear. Cancer cells are very sensitive to temperature changes compared to other cells. Therefore, this feature has created a new method called Hyperthermia for the treatment of cancer. In this method, the temperature of the cancerous tissue is increased to approximately $42^{\circ} \mathrm{C}$ for an appropriate period of time. ${ }^{38}$ This method causes the cancer cell to get disturbed and destroyed. In this method, with the increase in temperature, the blood vessels adjacent to the cancerous tissue are destroyed. By using ultrasound, radio waves and microwave, the temperature can be increased. This method, unlike X-rays and gamma, does not harm the body. In some cases, this procedure has side effects such as burns, swelling, pain and bleeding (Figure 8). This method is done with two external and internal techniques. In the external technique, highenergy waves are transmitted through the device to the tissue. But in the internal technique, a catheter enters cancerous tissue and the waves are released from the catheter. ${ }^{39,40}$

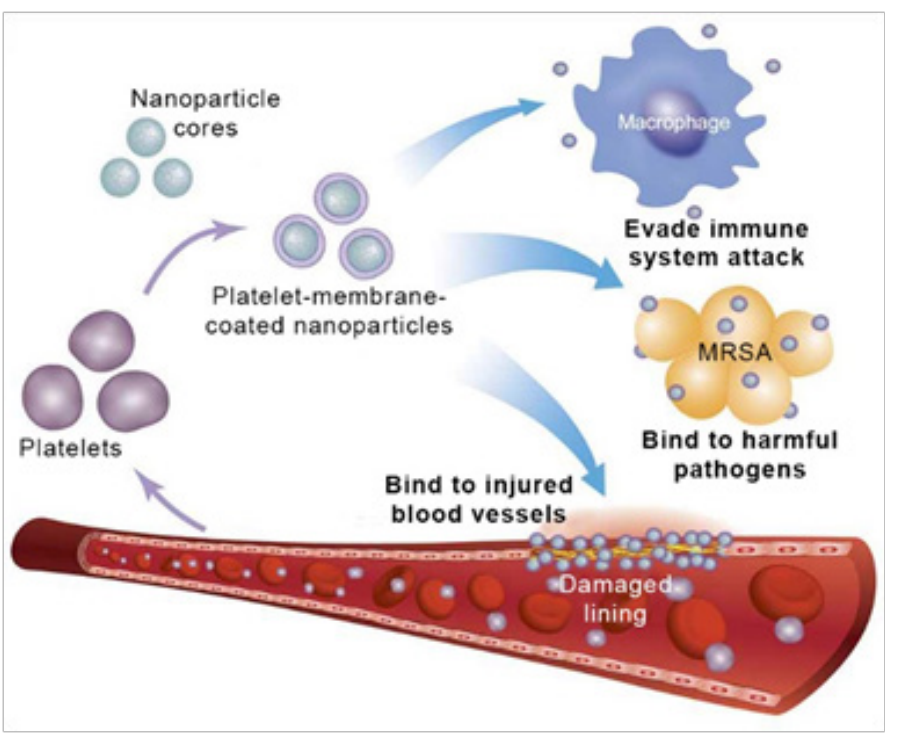

Figure 7 Targeting in the Drug Delivery System. ${ }^{37}$

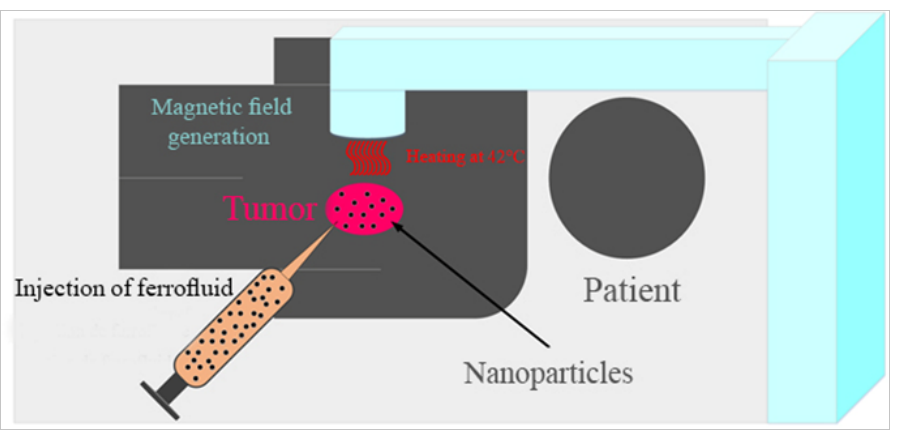

Figure 8 Hyperthermia method for the treatment of cancer.$^{40}$

\section{Results}

As mentioned, cancer is one of the most important causes of mortality among humans. Cancer is due to uncontrollable changes in the cells of the body. Breast and cervical cancer in women and prostate and gastric cancer in men are very common. Various ways to treat cancer include surgery, radiation therapy and chemotherapy. These methods can be done individually or done together. One of the important steps in radiation therapy is the treatment design based on the type of cancer and patient's condition. A simulation of the tumor position is performed to provide accurate radiation therapy. New ways to treat cancer have been developed, each with its own advantages and disadvantages. In the field of cancer treatment, researchers are more likely to look at ways in which cancer cells are more likely to be harmed, while healthy cells get the least impact. The types of cancer treatments mentioned in this article are summarized in Table 1. 
Table I Types and function of cancer treatment methods

\begin{tabular}{ll}
\hline Types of Cancer Treatment Methods & Function of Cancer Treatment Methods \\
\hline External Radiotherapy & Radiation of ionizing radiation outside the body \\
Brachytherapy & The placement of radioactive substances in the body temporarily or permanently \\
Immuno-oncology & Strengthening and stimulating the immune system to destroying tumors and cancer cells \\
Epigenetic & Changes in gene and DNA function \\
Drug Delivery & Placing drugs on magnetic nanoparticles and directing them to cancer cells \\
Hyperthermia & Increasing the temperature of the cancerous tissue to disorder the activity of the cancerous cell
\end{tabular}

\section{Discussion}

In the treatment of cancer, the DNA of the cancer cell is damaged and destroyed, and the cancer cells cannot reproduce or completely disappear. Radiation therapy using charged particles or high-energy radiation causes the cancer cell to be destroyed, but it also affects healthy cells. Radiation therapy is divided into two general types of external and internal, each with a different technique. In the radiation therapy using various methods, a simulation of the treatment is performed, which was previously done in two dimensions, but now it is done using three-dimensional methods. Radiation therapy has several disadvantages that researchers are looking for more efficient and less risky ways to treat cancer. In new methods, Immuno-oncology is used to treat cancer, which strengthens the patient's immune system. The Epigenetic technique changes the gene and DNA function of the cancer cell. The drug delivery method is one of the best ways to treat cancer that the cancer treatment drug directly enters into cancer cells and has no effect on other tissues. The Hyperthermia method, with changes in cancer cells temperature, disrupts the mechanism of these cells and destroys these cells.

\section{Conclusion}

Cancer is known as a deadly disease, an issue that is important in today's society. Therefore, researchers are researching cancer treatment to find better ways to treat cancer. Air pollution, inappropriate nutrition, Immobility, obesity, stress and harmful sun rays are important factors in the incidence of cancer, and about $10 \%$ of the cancers are genetic and hereditary. In the treatment of cancer, surgery, chemotherapy and radiotherapy are used, each with dangers and benefits. Radiation therapy uses high-energy ionizing radiation that affects cancer cells and healthy cells. This method is very dangerous for children and pregnant women. In new ways to treat cancer, the least harm is inflicted on healthy cells and most damage to cancer cells is applied. With different methods such as exercise, proper nutrition and health care, can be almost prevent cancer. In this paper, new methods for treating cancer were reviewed. Methods that only destroy cancerous cells and cause the least harm to healthy cells. Different methods of radiotherapy and their advantages and disadvantages have been investigated. Radiotherapy is done internally and externally, each of which is specially for the treatment of several types of cancers.

\section{Acknowledgements}

None

\section{Conflict of interests}

The author declares that they have no conflict of interest.

\section{References}

1. Coller HA. Is Cancer a Metabolic Disease? Am J Pathol. 2014;184(1):417.

2. Asthana S, Chauhan S, Labani S. Breast and cervical cancer risk in India: an Update. Indian J Public Health. 2014;58(1):5-10.

3. Shirzadfar H, Riahi S, Ghaziasgar MS. Cancer imaging and brain tumor diagnosis. Journal of Bioanalysis \& Biomedicine. 2017;9(1).

4. Blackadar CB. Historical review of the causes of cancer. World J Clin Oncol. 2016;7(1):54-86.

5. Zou X, Yao M, Ma L, et al. X-ray-induced nanoparticle-based photodynamic therapy of cancer. Nanomedicine. 2014;9(15): 2339-2351.

6. Aluwini S, Van Rooij P, Kirkels WJ, et al. Bladder function preservation with brachytherapy, external beam radiation therapy, and limited surger in bladder cancer patients: long-term results. Int J Radiat Oncol Biol Phys. 2014;88(3):611-617.

7. Shirzadfar H, Khanahmadi. Physical Principles and Recent Advances of Medical Imaging Systems. AASCIT Journal of Health. 2018;5(1):21-27.

8. Dearnaley DP. Escalated-dose versus control-dose conformal radiotherapy for prostate cancer: long-term results from the MRC RT01 randomised controlled trial. The Lancet Oncology. 2014;15(4):464-473.

9. Axente M, Paidi A, Von Eyben R, et al. Clinical evaluation of the iterative metal artifact reduction algorithm for CT simulation in radiotherapy. Medical Physics. 2015;42(3):1170-1183.

10. Huang M, Zhu H, Liu T, et al. Comparison of external radiotherapy and percutaneous vertebroplasty for spinal metastasis. Asia Pac J Clin Oncol. 2016;12(2):201-208.

11. Mohamed S, Kallehauge J, Fokdal L, et al. Parametrial boosting in locally advanced cervical cancer: Combined intracavitary/interstitial brachytherapy vs. intracavitary brachytherapy plus external beam radiotherapy. Brachytherapy. 2014;14(1):23-28.

12. Demanes DJ, Ghilezan MI. High-dose-rate brachytherapy as monotherapy for prostate cancer. Brachytherapy An International Multidisciplinary Journal. 2014;13(6):529-541.

13. Illidge T. Modern Radiation Therapy for nodal non hodgkin lymphomadtarget definition and dose guidelines from the international lymphoma radiation oncology group. Int J Radiat Oncol Biol Phys. 2014;89(1):49-58.

14. http://www.prostate.org.au/media/465106/hdr-brachytherapy.png

15. Krayenbuehl J, Norton I, Studer G, et al. Evaluation of an automated knowledge based treatment planning system for head and neck. Radiation Oncology. 2015;10(226):1-8.

16. Paulson ES, Erickson B, Schultz C, et al. Comprehensive MRI simulation methodology using a dedicated MRI scanner in radiation oncology for external beam radiation treatment planning. Medical Physics. 2015;42(1):28-39. 
17. Ling TC, Slater JM, Nookala P, et al. Analysis of intensity-modulated radiation therapy (IMRT), proton and 3D conformal radiotherapy (3DCRT) for reducing perioperative cardiopulmonary complications in esophageal cancer patients. Journal cancers. 2014;6(4):2356-2368.

18. Zhang B, Mo Z, Du W, et al. Intensity-modulated radiation therapy versus 2D-RT or 3D-CRT for the treatment of nasopharyngeal carcinoma:A systematic review and meta-analysis. Oral Oncology. 2015;51(11):1041-1046.

19. https://www.researchgate.net/profile/Luigi Raffaele/ publication/305415946/figure/fig1/AS:385825666813953@14689994 15982/Radiotherapy-plan-Radiotherapy-dose-distribution-in-axialcoronal-and-sagittal-views.jpg

20. Marta GN, Silva V, Carvalho HA, et al. Intensity-modulated radiation therapy for head and neck cancer: Systematic review and meta-analysis. Radiother Oncol. 2014;110(1):9-15.

21. Yu JB, Cramer LD, Herrin J, et al. Stereotactic body radiation therapy versus intensity-modulated radiation therapy for prostate cancer:comparison of toxicity. Journal of Clinical Oncology. 2014;32(12):1195-1201.

22. http://danwin.com/words/wp-content/uploads/2010/01/nyt-imrtgraphic.jpg

23. http://www.medic.usm.my/ department/jpnro/images/IMRTbiLat.gif

24. Nieder C, Grosu AL, Gaspar LE. Stereotactic radiosurgery (SRS) for brain metastases: a systematic review. Radiation Oncology. 2014;9(155):1-9.

25. Yamamoto M. Stereotactic radiosurgery for patients with multiple brain metastases (JLGK0901):a multi-institutional prospective observational study. Lancet Oncology. 2014;15(4):387-395.

26. http://s.enterocms.com/cache/1/AEXdkcF 2 y 8 n 9 ne R 9 Z c u 39 q y Y c 2 G Y M 2.jpg ? f i t $=$ c r o p center $\& w=780 \& s=22 \mathrm{fd} 32$ ee 66 bada5b4b5c0915f0d06bdc

27. http://face-facts.org/wp-content/uploads/2016/12/Gammaknife.jpeg

28. Fogarty GB, Hong A, Scolyer RA, et al. Radiotherapy for lentigo maligna - a literature review and recommendations for treatment. Br J Dermatol. 2014;170(1):52-58.
29. Simpson BM, Foster SK, Chapman CN, et al. The effectiveness of grenz ray therapy for chronic dermatoses of the hands and feet. Dermatitis. 2014;25(4):205-208.

30. Healy BJ, Merwe D, Christaki KE, et al. Cobalt-60 Machines and medical linear accelerators: competing technologies for external beam radiotherapy. Clinical Oncology. 2016;29(2):110-115.

31. Merna C, Rwigema JC, Cao M, et al. A treatment planning comparison between modulated tri-cobalt-60 teletherapy and linearacceleratorbased stereotactic body radiotherapy for central early-stagenon small cell lung cancer. Med Dosim. 2016;41(1):87-91.

32. Taylor CW, Kirby AM. Cardiac side-effects from breast cancer radiotherapy. Clinical Oncology. 2015;27(11):621-629.

33. Kohrt HE, Tumeh PC, Benson D, et al. Immunodynamics: a cancer immunotherapy trials network review of immune monitoring in immunooncology clinical trials. J Immunother Cancer. 2016;4(15):1-16.

34. Wouters BJ, Delwel R. Epigenetics and approaches to targeted epigenetic therapy in acute myeloid leukemia. Blood journal. 2016;127(1):42-52.

35. Sun T, Zhang YS, Pang B, et al. Engineered nanoparticles for drug delivery in cancer therapy. Angew Chem Int Ed Engl. 2014;53(46):12320-12364.

36. Taheri-Kafrani A, Shirzadfar H, Tavassoli-Kafrani A. Dendrimers and dendrimers-grafted superparamagnetic iron oxide nanoparticles:synthesis, characterization, functionalization, and biological applications in drug delivery systems. Nano- and Microscale Drug Delivery Systems. 2017; Chapter 5:75-91.

37. https://3c1703fe8d.site.internapcdn.net/newman/gfx/news/hires/2015 targeteddrug.jpg

38. Shirzadfar H, Nadi M, Kourtiche D, et al. Needle-type GMR sensor to estimate the magnetic properties of diluted ferrofluid for biomedicine application. IRBM. 2015;36(3):178-184.

39. Toraya-Brown S, Fiering S. Local tumour hyperthermia as immunotherapy for metastatic cancer. Int J Hyperthermia. 2014;30(8):531-539.

40. Shirzadfar H. Conception et réalisation d'un biocapteur à GMR pour la caractérisation de milieux biologiques. Doctoral dissertation, Université de Lorraine; 2014 